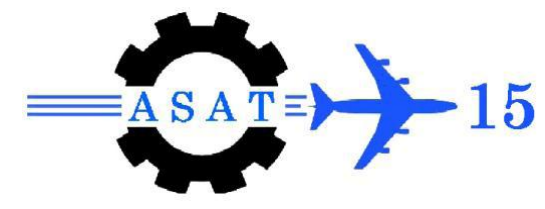

\title{
Simulation Study of 3D Polarization Independent Fishnet Negative Index Metamaterials at $40 \mathrm{GHz}$
}

\author{
M. A. Nafe ${ }^{*}$, W. S. Zaoui ${ }^{\dagger}$, M. Berroth ${ }^{\ddagger}$
}

\begin{abstract}
Superlensing is an important negative index metamaterial application that extends from modern biomedical fields to high-resolution radar systems for sub-wavelength imaging. In this work, a rigorous simulation study of a 3D polarization independent negative index metamaterial structure has been carried out to investigate its use as a superlens in the microwave region at around $40 \mathrm{GHz}$. Ten weakly and strongly coupled cross-cylinder fishnet negative index metamaterial layers have been investigated aiming to find a suitable optimized structure to be used as a superlens with a resolution overcoming the half wavelength diffraction limit in the microwave frequency range, especially at around $40 \mathrm{GHz}$. This study shows that ten weakly coupled layers of optimized cross-cylinder negative index fishnet structure have the advantage of fast convergence of their effective medium parameters. The spacer between consecutive unit cells causes the structure to be mismatched with free space. This results in high reflections and hence it is not suitable to be used as a microwave superlens. When the spacer between consecutive layers is removed the convergence is not as fast as in the previous case but good matching with free space occurs. The figure of merit of the ten strongly coupled cross-cylinder fishnet structure is enormously increased to a peak value of 45 at $39 \mathrm{GHz}$ and the focusing capability of this structure in a double slit configuration shows sub-wavelength resolution.
\end{abstract}

Keywords: Metamaterial, negative refractive index, fishnet, superlensing.

\section{Introduction}

Conventional lenses, no matter how powerful, are only able to magnify light to nearly half of the wavelength, or the so-called diffraction limit. Practically this limits the resolution of all lithography and imaging systems. Moreover, the dimensions of optical storing devices or the size of the features created on silicon chips are limited to how much focusing power a particular lens has [1-3].

Superlensing utilizing a left-handed metamaterial slab allows "perfect imaging" if it is completely lossless, impedance matching, and its refractive index is $n=-1$. Under these conditions, the superlens, called the perfect lens, would focus the entire spectrum with resolution overcoming the diffraction limit; both propagating as well as evanescent spectra. This is considered as an advantage over conventional lenses [4].

* German University in Cairo, Faculty of Information Engineering and Technology, Main Entrance Al Tagamoa Al Khames, 13411 Cairo, Egypt; mahmoudalinafe@gmail.com .

$\dagger \quad$ University of Stuttgart, Institute of Electrical and Optical Communications Engineering, Pfaffenwaldring 47, 70569 Stuttgart, Germany; wissem.sfarzaoui@int.uni-stuttgart.de .

$\$$ University of Stuttgart, Institute of Electrical and Optical Communications Engineering, Pfaffenwaldring 47, 70569 Stuttgart, Germany. 
The fishnet structure based on a metal-substrate-metal composition is so far the best design to give a negative refractive index behavior at high frequencies due to its simplicity in fabrication, ability to produce left handed (LH) behavior for normal incidence and appropriateness for two- and three-dimensional applications [5]. In the present simulation work, 3D metamaterial structures are achieved by stacking ten fishnet layers periodically along the direction of propagation. Each single layer consists of an identical unit cell of a cross-cylinder fishnet structure arranged periodically in two dimensions perpendicular to the direction of propagation.

The used unit cell is a combination of a cross and a cylinder, which introduces a new parameter R (Fig. 1) to the well-known cross fishnet design. This parameter plays an important role in controlling the normalized impedance $\mathrm{z}$ to obtain a better matching to free space. This novel design was simulated and tested experimentally by W. Sfar Zaoui et al. for a single layer in the Q-band [6]. It showed superior behavior compared to the well-known cross design and provided good impedance matching to free space in a large frequency range and thus high transmission and low reflection properties.

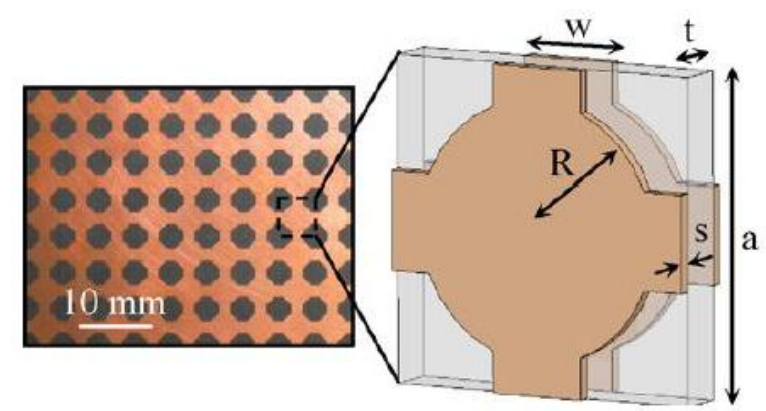

Fig. 1: Cross-cylinder single layer fishnet metamaterial structure investigated by W. Sfar Zaoui et al. [6].

All numerical simulations are done using the finite integration solver Computer Simulation Technology (CST Microwave Studio). A theoretical model at normal incidence is developed based on an artificial waveguide in front and behind the unit cell with two ideal magnetic conductor planes and two ideal electric conductor planes as the transverse boundaries. It is equivalent to an infinite layer medium with a linear polarized electromagnetic plane wave. To reduce the computing time and expenses, only a quarter of the structure is simulated since it has two planes of symmetry. As the simulated metamaterial structures are composed of unit cells with thickness smaller than the wavelength $\lambda$ at the target frequency $40 \mathrm{GHz}$ in the direction of propagation, the electromagnetic waves do not resolve the details of the structure. Thus, the effective material parameters (EPs) are obtained by applying the effective medium theory [7].

In the present study, ten weakly coupled layers of cross-cylinder polarization independent fishnet structures are investigated and optimized. To overcome the spacer losses, a second design of ten strongly coupled layers is studied. For a closer investigation of the negative refractive index (NRI) behavior, a wedge simulation is performed where the negative refraction phenomenon is observed. Finally, the sub-wavelength resolution capability of the optimized design is investigated numerically by obtaining the near-field distribution of a two slit object resolved by the 3D strongly coupled layers [8]. 


\section{Simulation Study of the Weakly Coupled Polarization Independent Cross-Cylinder Fishnet Metamaterial Structure}

The unit cell of the studied cross-cylinder fishnet structure is shown is Fig. 2a. The size of the unit cell along the direction of propagation $a_{z}$ is defined as $a_{z}=d+2 t+s$ where $d, t$ and $s$ are the distance between two consecutive fishnet layers, the dielectric and the metal thickness, respectively. Consecutive layers are separated by an air gap of $0.5 \mathrm{~mm}$. The substrate material is Duroid 5880 with a thickness $\mathrm{t}=250 \mu \mathrm{m}$, a dielectric constant $\varepsilon_{\mathrm{r}}=2.2$ and a very low tangent loss $\tan \delta=0.0009$ at $40 \mathrm{GHz}$. Each of the copper plates have a thickness $\mathrm{s}=17.5 \mu \mathrm{m}$. Thus, the total thickness of the unit cell along the direction of propagation is $0.785 \mathrm{~mm}$. The optimized values for the parameters of the proposed unit cell are $\mathrm{R}=2 \mathrm{~mm}$, $\mathrm{w}=1.8 \mathrm{~mm}$ and $\mathrm{a}=4.8 \mathrm{~mm}$. Fig. $2 \mathrm{~b}$ depicts the three dimensional simulated ten weakly coupled cross-cylinder polarization independent metamaterial layers with a total thickness of $7.85 \mathrm{~mm}$.

a)

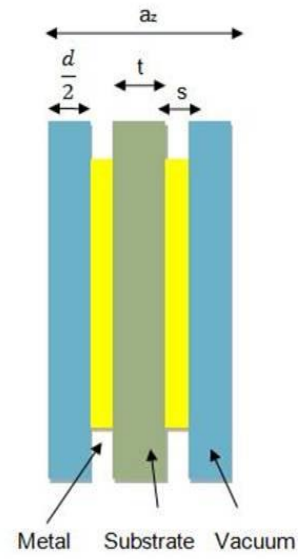

b)

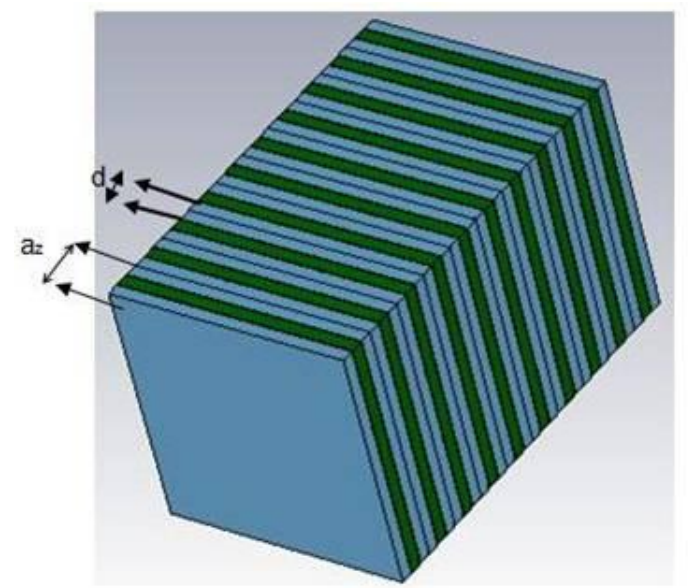

Fig. 2: (a) Unit cell parameters and (b) ten weakly coupled layers of the polarization independent cross-cylinder fishnet structure.

The amplitude and phase of the S-parameters of the simulated ten weakly coupled layers are depicted in Fig 3. The spectrum has a maximum transmission peak of $\left|\mathrm{S}_{21}\right|=-5.2 \mathrm{~dB}$ at 38.34 GHz. At this frequency, the reflection is $\left|S_{11}\right|=-10.1 \mathrm{~dB}$. The jump in the phase of $S_{21}$ is due to the sign change of the refractive index to a negative value [9].

a)

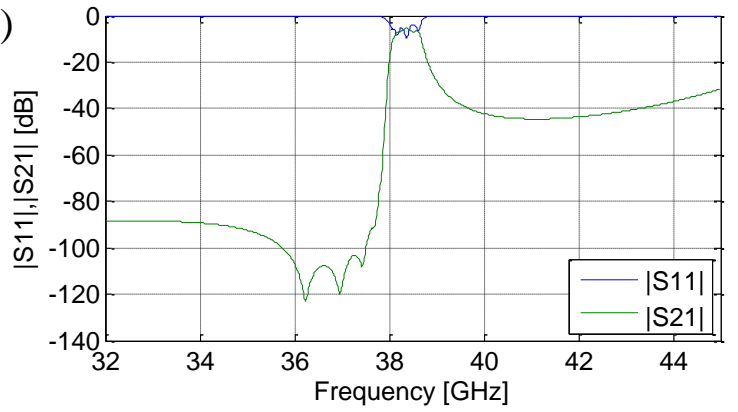

b)

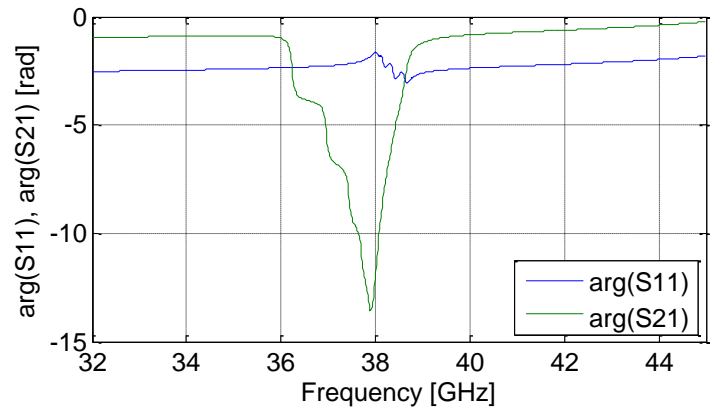

Fig. 3: Simulated S-parameters (a) amplitude and (b) phase spectra of the ten weakly coupled layers. 
The effective constitutive parameters ( $n, z, \mu$ and $\varepsilon$ ) are retrieved using the calculated $\mathrm{S}$-parameters. Due to symmetry, the problem is simplified since

$$
\mathrm{S}_{11}=\mathrm{S}_{22}, \mathrm{~S}_{12}=\mathrm{S}_{21}
$$

For a plane wave normally incident on a homogenous slab with thickness $d$ and with the first face of the slab considered as the origin, $S_{11}$ is equal to the reflection coefficient while $S_{21}$ is related to the transmission coefficient $\mathrm{T}$ by

$$
\mathrm{S}_{21}=\mathrm{Te}^{-\mathrm{jk} \mathrm{o}_{\mathrm{o}}}
$$

where $\mathrm{k}_{0}$ is the wave number of the incident wave in free space. The S-parameters are related to the refractive index $\mathrm{n}$ and the impedance $\mathrm{z}$ by

$$
\begin{gathered}
S_{11}=\frac{r\left(1-e^{-j 2 n k_{o} d}\right)}{1-r^{2} e^{-j 2 n k_{o} d}} \\
S_{21}=\frac{\left(1-r^{2}\right) e^{-j n k_{o} d}}{1-\left(r^{2} e^{-j n k_{o} d}\right)} \\
r=\frac{z-1}{z+1}
\end{gathered}
$$

where $\mathrm{z}$ is the normalized impedance, i.e. the ratio between the wave impedance in the medium $\mathrm{Z}$ to the wave impendence in vacuum $\mathrm{Z}_{0}$.

$$
\mathrm{z}=\frac{\mathrm{z}}{\mathrm{z}_{0}}
$$

The refractive index $\mathrm{n}$ and the normalized impedance $\mathrm{z}$ are obtained by inverting and solving equations (3) and (4), yielding

$$
\mathrm{z}=\mp \sqrt{\frac{\left(1+\mathrm{S}_{11}\right)^{2}-\mathrm{S}_{21}^{2}}{\left(1-\mathrm{S}_{11}\right)^{2}-\mathrm{S}_{21}{ }^{2}}}
$$

where

$$
\mathrm{e}^{-\mathrm{jnk}_{\mathrm{o}} \mathrm{d}}=\mathrm{X} \mp \sqrt{1-\mathrm{X}^{2}}
$$

and

$$
X=\frac{1}{2 S_{21}\left(1-S_{11}{ }^{2}+S_{21}{ }^{2}\right)} .
$$

Since the homogenous slab is a passive medium, the signs in equations (7) and (8) are determined by the requirement that

$$
\operatorname{Re}(\mathrm{z}) \geq 0
$$

and

$$
\operatorname{Im}(\mathrm{n}) \geq 0 \text {. }
$$

The value of the refractive index $n$ can be determined from equation (8) as 


$$
\mathrm{n}=\frac{1}{\mathrm{k}_{\mathrm{o}} \mathrm{d}}\left\{\left(\operatorname{Im}\left(\ln \left(\mathrm{e}^{-\mathrm{jnk} \mathrm{d}}\right)\right)+2 \mathrm{~m} \pi\right)-\mathrm{j} \operatorname{Re}\left(\ln \left(\mathrm{e}^{-\mathrm{jnk} \mathrm{o}_{\mathrm{o}} \mathrm{d}}\right)\right)\right\} .
$$

The imaginary part of $n$ is uniquely determined. However, the real part of $n$ is ambiguous due to the branches of the logarithmic function, where $\mathrm{m}$ is an integer indicating the branch index. To get the correct value, the continuity of the refractive index must be enforced. The permittivity $\varepsilon$ and the permeability $\mu$ are then directly calculated from

$$
\varepsilon=\frac{\mathrm{n}}{\mathrm{z}}, \mu=\mathrm{nz} \text {. }
$$

The retrieved relative impedance $\mathrm{z}$, refractive index $\mathrm{n}$, effective permittivity $\varepsilon$ and effective permeability $\mu$ are illustrated in Fig.4. At $38.34 \mathrm{GHz}$ the relative impedance is $\mathrm{z}=0.63-0.33 \mathrm{j}$. The relative refractive index $\mathrm{n}$, whose real part is negative all over the range from $36 \mathrm{GHz}$ till $38.77 \mathrm{GHz}$, is consistent with the phase jump of $\mathrm{S}_{21}$ in Fig. 3. At $38.34 \mathrm{GHz}$ the refractive index is $\mathrm{n}=-0.87+0.1 \mathrm{j}$. The effective permittivity is negative until the effective plasma frequency $46 \mathrm{GHz}$, while the effective permeability has a magnetic resonance at around $38 \mathrm{GHz}$ and its real part is negative between $38 \mathrm{GHz}$ and $38.75 \mathrm{GHz}$. Thus, the structure shows a double negative behavior within the frequency range $38 \mathrm{GHz}$ till $38.75 \mathrm{GHz}$. At $38.34 \mathrm{GHz} \varepsilon=-1.14-0.64 \mathrm{j}$ and $\mu=-0.51+0.35 \mathrm{j}$.

a)

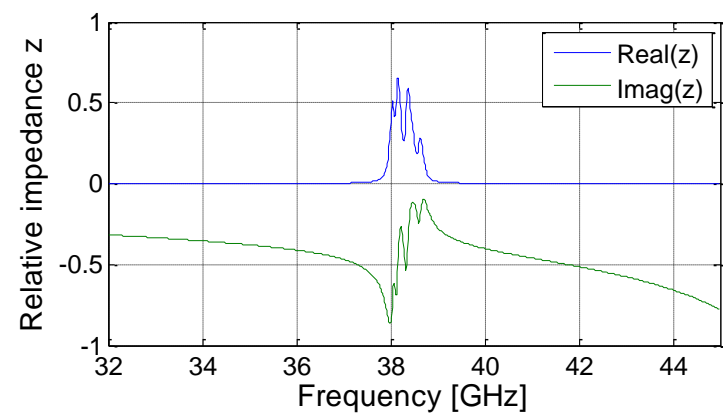

c)

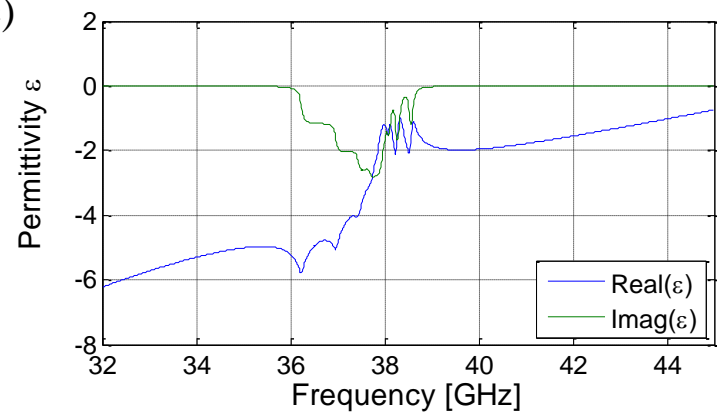

b)

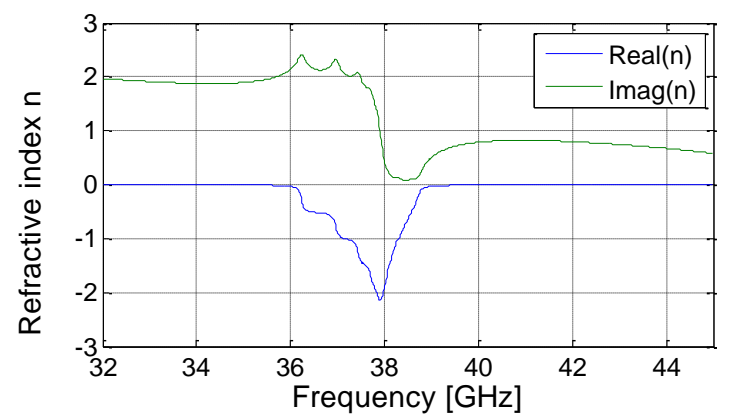

d)

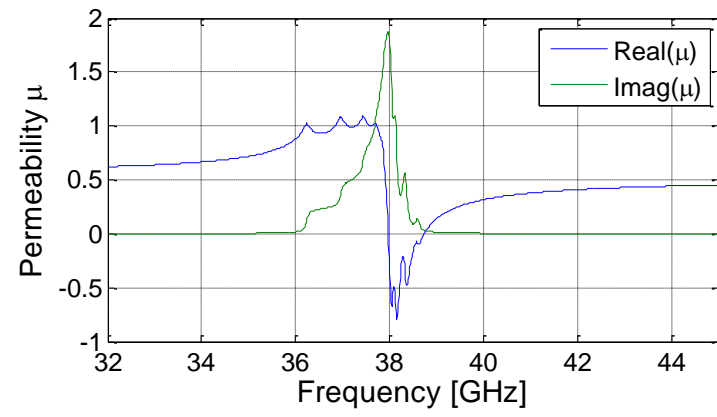

Fig. 4: Retrieved (a) relative impedance, (b) refractive index, (c) effective permittivity and (d) effective permeability of the ten weakly coupled layers.

The quality of the metamaterial is defined by the figure of merit, which is the ratio of the real part to the imaginary part of the retrieved effective refractive index $(\mathrm{FOM}=\operatorname{Re}(\mathrm{n}) / \mathrm{Im}(\mathrm{n}))$. The figure of merit for the ten weakly coupled layers is shown in Fig. 5. It is considered as the factor to measure the transmission loss and indicates the quality of the negative refraction [10]. The low transmission of the propagating waves is due to the spacer losses. Since the distance between the layers is large, i.e. the coupling between the layers is weak, the losses dominate the structure. The FOM has a low value $(<10)$ throughout the whole frequency range which explains the low transmission. At $38.34 \mathrm{GHz}$ the FOM reaches a value of 9.09 and a peak value of 9.34 at $38.38 \mathrm{GHz}$. 
The convergence of the retrieved effective medium parameters is studied by simulating the propagating modes with CST Microwave Studio eigenmode solver. Infinite number of layers along the direction of propagation is assumed and the corresponding phase shift is swept. The dispersion diagram $\omega(\beta)$, shown in Fig.6a, is obtained. For the first mode, the fundamental mode, as the frequency increases, the phase decreases. This negative slope of the phase in the frequency range $38 \mathrm{GHz}-38.81 \mathrm{GHz}$ indicates that the structure shows a NRI behavior. The NRI frequency range obtained from the eigenmode solver fits the NRI frequency range obtained by the frequency-domain simulations.

The real part of the refractive index of the fundamental mode for the infinitely periodic weakly coupled cross-cylinder fishnet unit cell is plotted against the real part of the refractive index of ten weakly coupled layers. As shown in Fig.6b, the two curves overlap well with each other, indicating the convergence of the retrieved effective medium parameters.

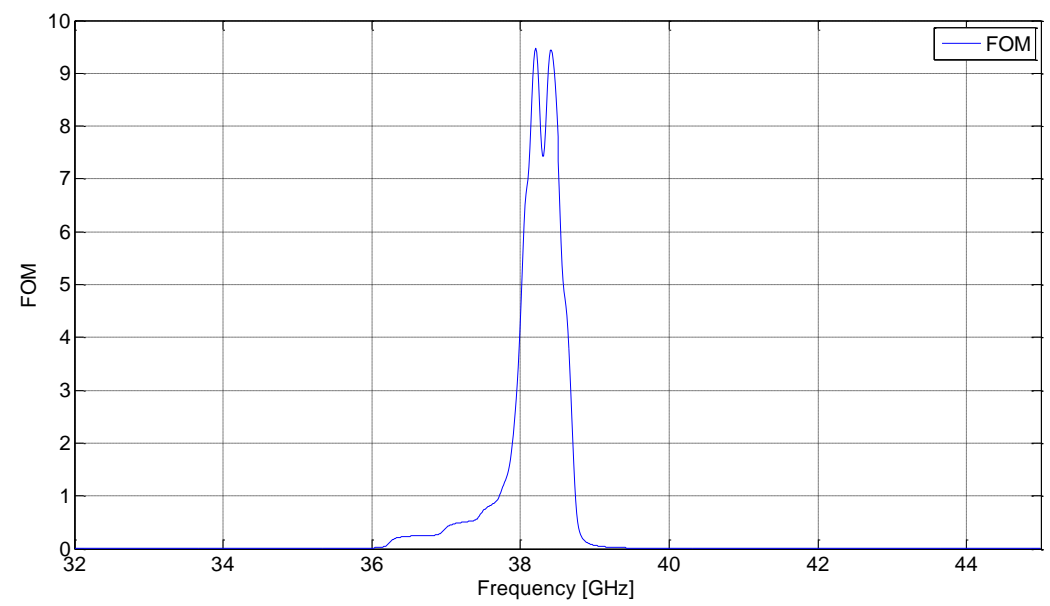

Fig. 5: Calculated figure of merit of the ten weakly coupled layers.

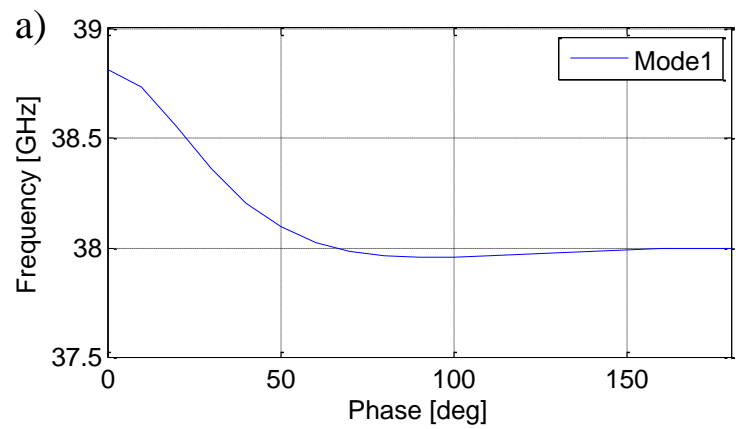

b)

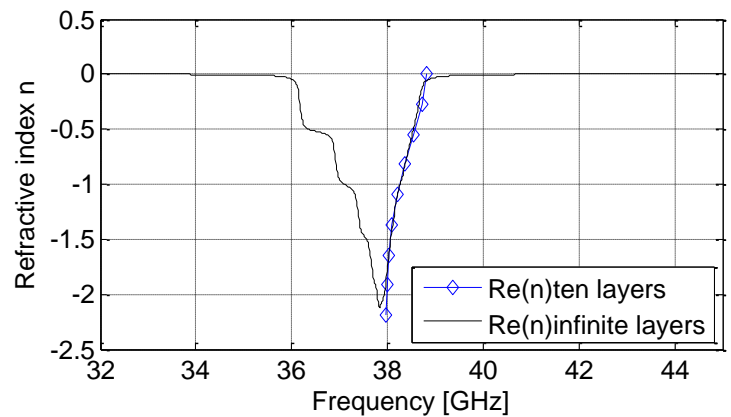

Fig. 6: (a) Dispersive relation of infinitely periodic weakly coupled unit cells, (b) refractive index of infinite number of layers against the refractive index of the ten weakly coupled layers.

\section{Simulation of the Strongly Coupled Polarization Independent Cross- Cylinder Fishnet Metamaterial Structure}

To overcome the transmission losses and high reflections, the coupling between the consecutive layers is increased by removing the spacing between the layers. The size of the unit cell along the direction of propagation $a_{z}$ is now defined as $a_{z}=2 t+s$. The fishnet unit cell is illustrated in Fig. 7a. The structure is printed on a $250 \mu \mathrm{m}$ thick Duroid 5880 dielectric substrate with a $17.5 \mu \mathrm{m}$ thick copper layer on both sides. The size of the unit cell along the direction of propagation is $0.285 \mathrm{~mm}$. Thus, the total thickness of the whole ten layers is 
$2.85 \mathrm{~mm}$ compared to $7.85 \mathrm{~mm}$ of the weakly coupled layers (Fig. 7b). The unit cell is repeated along the $x$ and $y$ directions with a periodicity of $a_{x}=a_{y}=a=4.9 \mathrm{~mm}$. The optimized values of the proposed unit cell parameters are $\mathrm{R}=2.3 \mathrm{~mm}$ and $\mathrm{w}=1.45 \mathrm{~mm}$.

a)

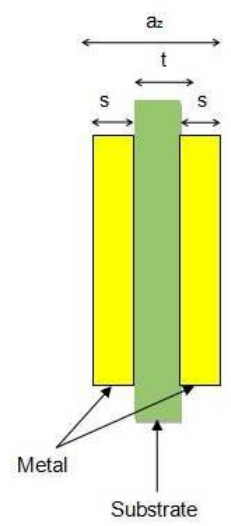

b)

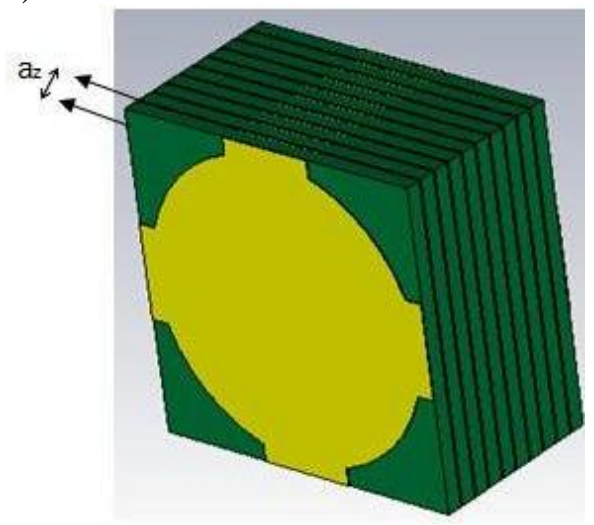

Fig. 7: (a) Unit cell parameters and (b) the total ten strongly coupled layers.

The amplitude and phase of the simulated S-parameters are depicted in Fig. 8a and Fig 8b. The transmission spectrum exhibits two band pass intervals. The first band between $38 \mathrm{GHz}$ and $40 \mathrm{GHz}$ corresponds to the negative refractive index behavior. The second band is between $40 \mathrm{GHz}$ and $43.3 \mathrm{GHz}$ and has a positive refractive index behavior. At $38.45 \mathrm{GHz}$, the transmission $\left|\mathrm{S}_{21}\right|$ is $-0.58 \mathrm{~dB}$ and the reflection $\left|\mathrm{S}_{11}\right|$ is $-24.8 \mathrm{~dB}$. The jump in the phase of $\mathrm{S}_{21}$ is due to the sign change of the refractive index to negative values [9].

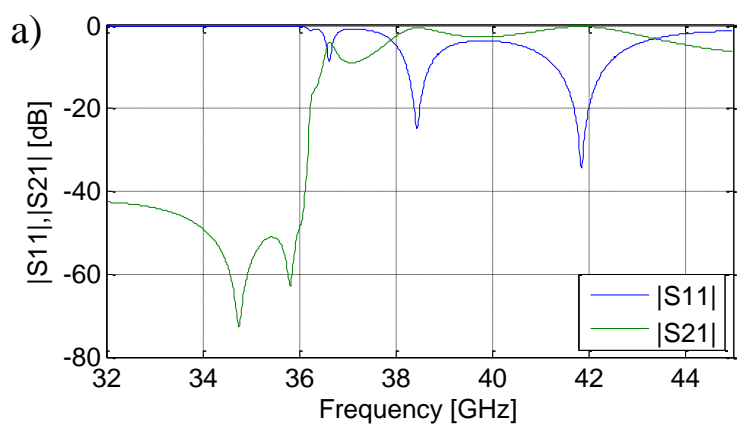

b)

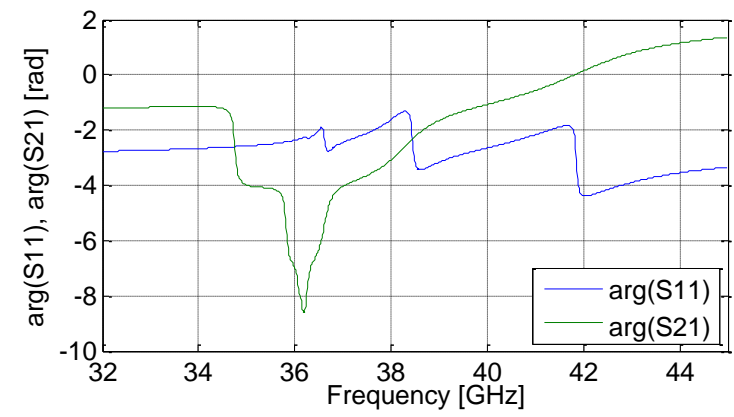

Fig. 8: (a) Amplitude and (b) phase spectra of the simulated S-parameters of the ten strongly coupled layers

As shown in Fig.9, the retrieved relative impedance at $38.45 \mathrm{GHz}$ is $\mathrm{z}=0.98-0.08 \mathrm{j}$. The relative refractive index $n$, whose real part is negative in a large frequency range of almost $7 \mathrm{GHz}$, is consistent with the phase jump. At $38.45 \mathrm{GHz}$ the refractive index is $\mathrm{n}=-1.02+0.03 \mathrm{j}$. The real part of the effective permittivity is negative until the effective plasma frequency $41.8 \mathrm{GHz}$, while the effective permeability has a magnetic resonance at around $38.1 \mathrm{GHz}$ and its real part is negative between around $38.1 \mathrm{GHz}$ and $41.6 \mathrm{GHz}$. As a result, the fishnet is double negative between $38.1 \mathrm{GHz}$ and $41.6 \mathrm{GHz}$ which corresponds to a large relative NIM bandwidth of $9 \%$. At $38.45 \mathrm{GHz}$ the effective permittivity and effective permeability are $\varepsilon=-1.03-0.06 \mathrm{j}$ and $\mu=-0.99+0.11 \mathrm{j}$, respectively. 
a)

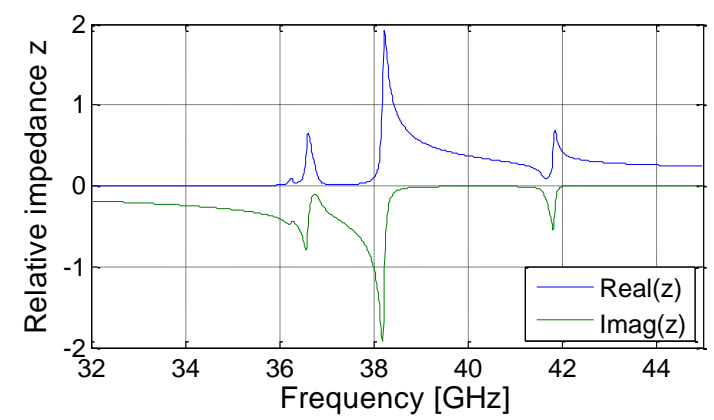

c)

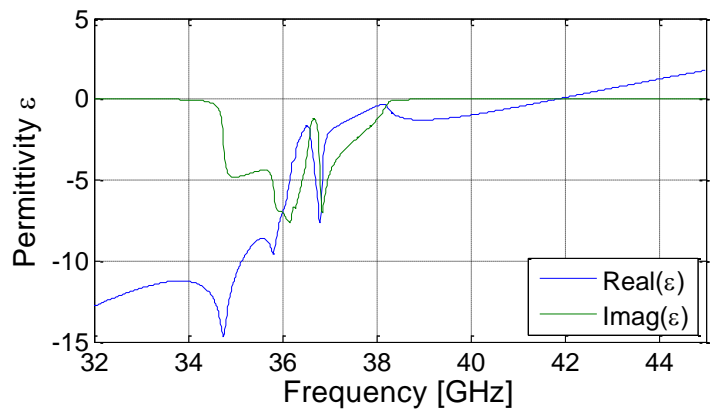

b)

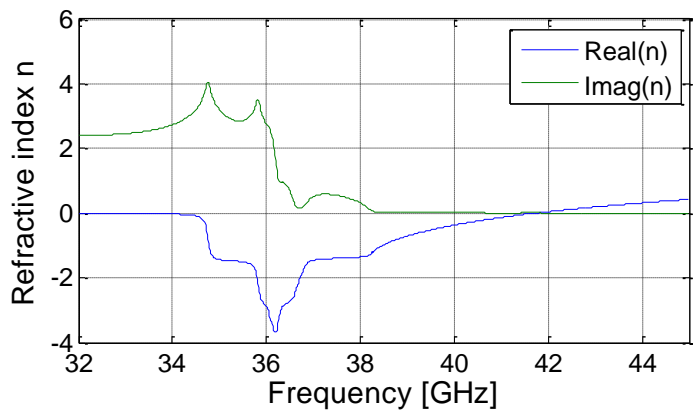

d)

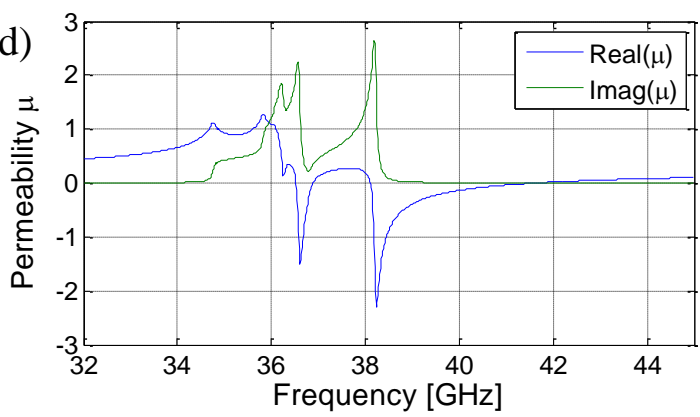

Fig. 9: Retrieved a) relative impedance, b) refractive index, c) effective permittivity and d) effective permeability.

The Figure of merit of the ten strongly coupled layers is depicted in Fig.10. The FOM reaches 34.68 at $38.45 \mathrm{GHz}$ and a peak value of 44.6 at $39 \mathrm{GHz}$. The obtained figure of merit of the $3 \mathrm{D}$ metamaterial is one of the highest FOMs reported in literature where values $<25$ are obtained [10,11]. Thus, the proposed ten strongly coupled cross-cylinder polarization independent fishnet structure is seen as an improvement over current reached results of $3 \mathrm{D}$ structures in nearby frequencies.

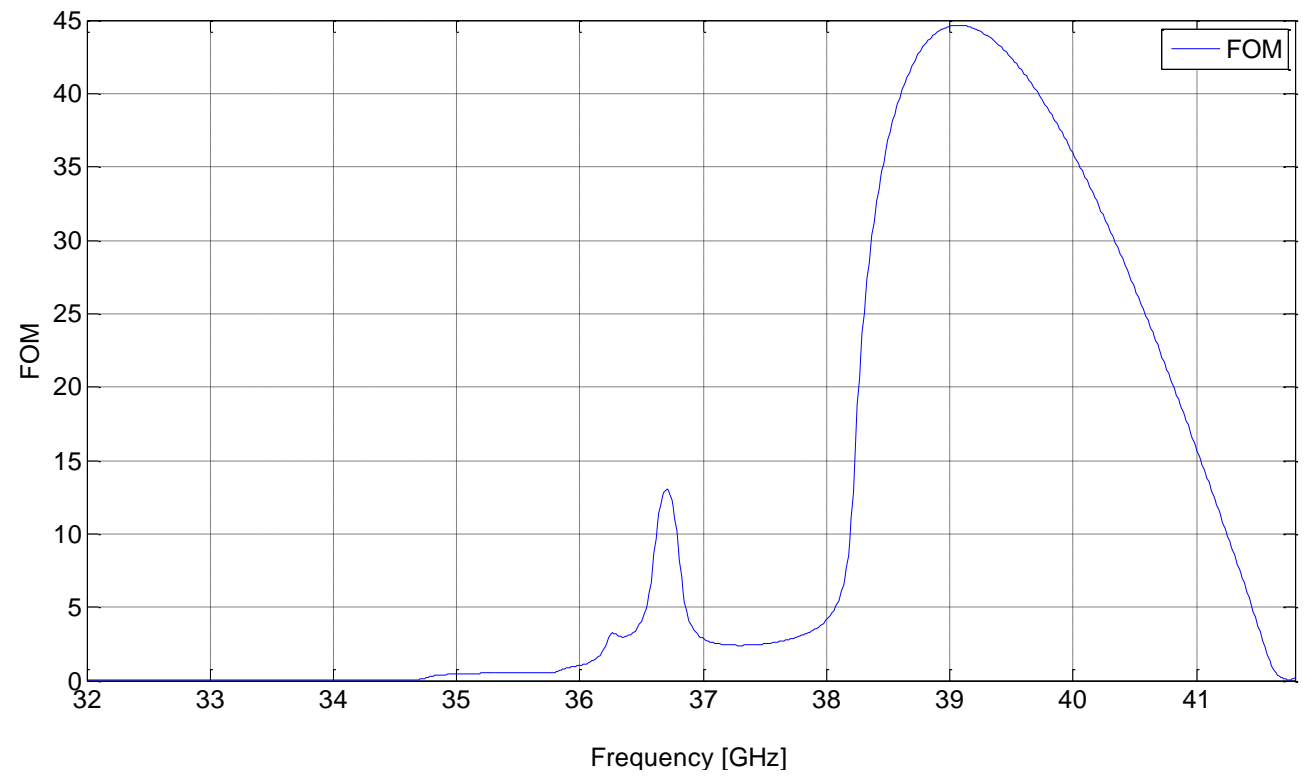

Fig. 10: Calculated figure of merit of the ten strongly coupled cross-cylinder fishnet structure. 
The convergence of the retrieved effective medium parameters, is shown in Fig.11a, where the dispersion diagram $\omega(\beta)$ for the first propagating mode is presented. The negative slope of the phase in the frequency range $36.26 \mathrm{GHz}-38.71 \mathrm{GHz}$ indicates that the structure shows a NRI behavior within this frequency region. The NRI frequency range obtained from the eigenmode solver fits the NRI frequency range obtained by the frequency-domain simulations.

The real part of the refractive index of the fundamental mode for the infinitely periodic strongly coupled cross-cylinder fishnet unit cell and the real part of the refractive index of the ten strongly coupled layers are plotted in Fig.11b. There is a significant difference between the two curves indicating that the retrieved effective medium parameters of the ten layers have not converged yet.

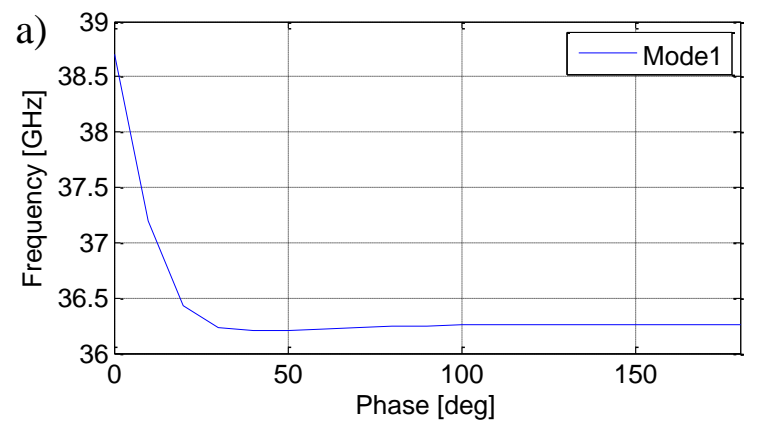

b)

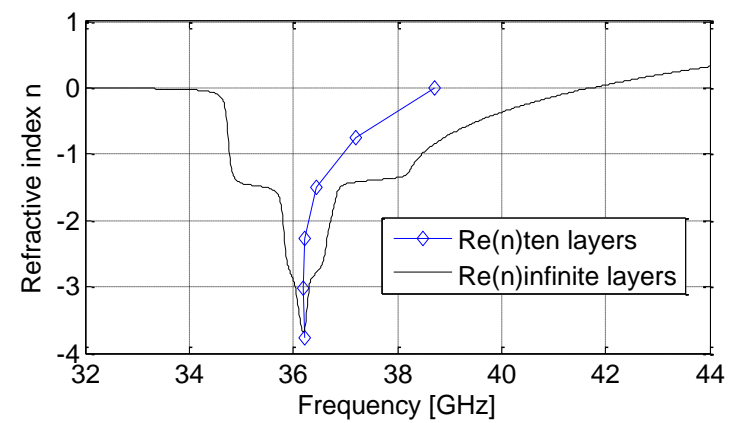

Fig. 11: (a) Dispersive relation of infinitely periodic strongly coupled unit cells, (b) refractive index of infinite number of layers against the refractive index of the ten strongly coupled layers.

\section{Wedge Simulation}

To closely investigate the NRI behavior of the strongly coupled unit cells, a wedge composed by stacking 19 to 2 layers from left to right making an inclination angle of $\alpha=3.3^{\circ}$ is simulated. The wedge has a height $\mathrm{H}=5.38 \mathrm{~mm}$ and a length $\mathrm{L}=93.1 \mathrm{~mm}$. The wedge is excited by a plane wave and guided by a perfectly electronic conducting (PEC) waveguide. The guided plane waves are faced by eleven, ten and nine strongly coupled unit cells from left to right.

At $38.45 \mathrm{GHz}$, the ten strongly coupled unit cells have a refractive index of $n=-1.019$. The EM waves are expected to refract negatively with an angle of $\theta=-3.36^{\circ}$ as depicted in Fig. 12. However, the refracted EM waves are toggling between $\theta=-3^{\circ}$ and $\theta=-3.6^{\circ}$. This toggling is due to the slow convergence of the retrieved refractive index of the strongly coupled unit cells. To study this toggling, nine and eleven strongly coupled unit cells are simulated. The change in the refractive index and the normalized impedance at $38.45 \mathrm{GHz}$ are shown in table 1. For nine unit cells, the real part of the refractive index decreases to $\mathrm{n}_{9}=-0.927$ and the wave impedance decreases to $\mathrm{z}_{9}=0.78$, while for eleven unit cells, the real part of the refractive index increase to $\mathrm{n}_{11}=-1.091$ and the wave impedance increases to $\mathrm{z}_{11}=1.34$. 


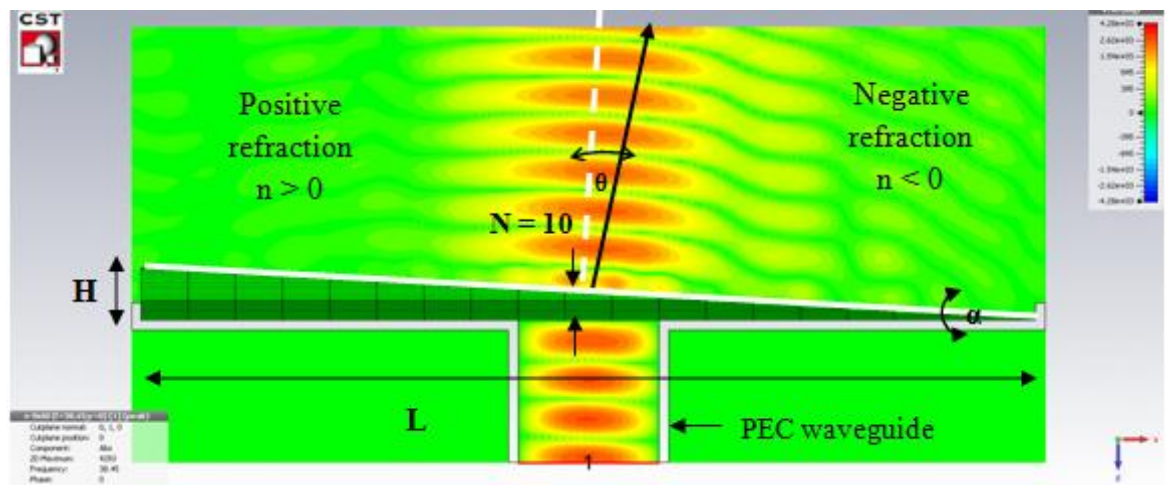

Fig. 12: Negative refraction of EM waves from a wedge formed by strongly coupled unit cells.

Table 1: Variation of the refractive index and the normalized impedance of nine, ten and eleven layers at $38.45 \mathrm{GHz}$

\begin{tabular}{ccc}
\hline \hline Number of layers & Refractive index & Normalized impedance \\
$\mathrm{N}$ & $\mathrm{n}$ & $\mathrm{z}$ \\
\hline \hline 9 & $-0.927+0.023 \mathrm{j}$ & $+0.78-0.034 \mathrm{j}$ \\
10 & $-1.019+0.029 \mathrm{j}$ & $+0.98-0.083 \mathrm{j}$ \\
11 & $-1.091+0.038 \mathrm{j}$ & $+1.34-0.169 \mathrm{j}$ \\
\hline \hline
\end{tabular}

\section{Double Slit Simulation}

The sub-wavelength capability of the 3D ten strongly coupled polarization independent crosscylinder fishnet metamaterial structure is found by evaluating the electric field across the image plane in a double slit configuration with a slit width $\mathrm{a}=4.9 \mathrm{~mm}$ and for different slit distance values. The double slit configuration consists of a plane wave source followed by a copper plate with two slits. The two slits act as two secondary wave sources according to Huygens principal. The metamaterial structure with thickness of $2.85 \mathrm{~mm}$ is then placed $1 \mathrm{~mm}$ away from the object plane along the propagation direction. The EM waves emanating from the two secondary sources are focused inside and outside the lens at the image plane as shown in Fig. 13.

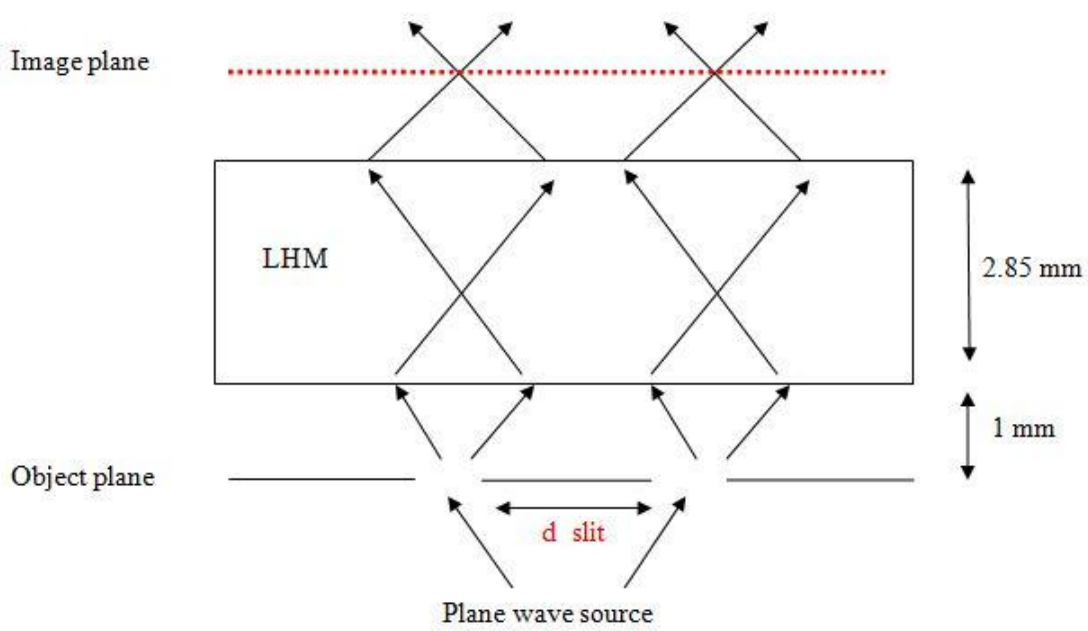

Fig. 13: Double slit simulation model 
The electric field is plotted against the distance along the image plane for different slit distance values from $\lambda / 2$ to $\lambda / 16$, as depicted in Fig.13. At a value of $\lambda / 2$, the two peaks of the image are clearly seen. These two peaks start to be less distinguishable when decreasing the slit distance and completely disappear at a value of $\lambda / 16$. The theoretical resolution is numerically calculated following the analytical method described in [8] and is determined as $\lambda / 3$. The resolution can be furthermore enhanced by decreasing the losses and reducing the thickness of the NIM, i.e. decreasing the number of layers [12].

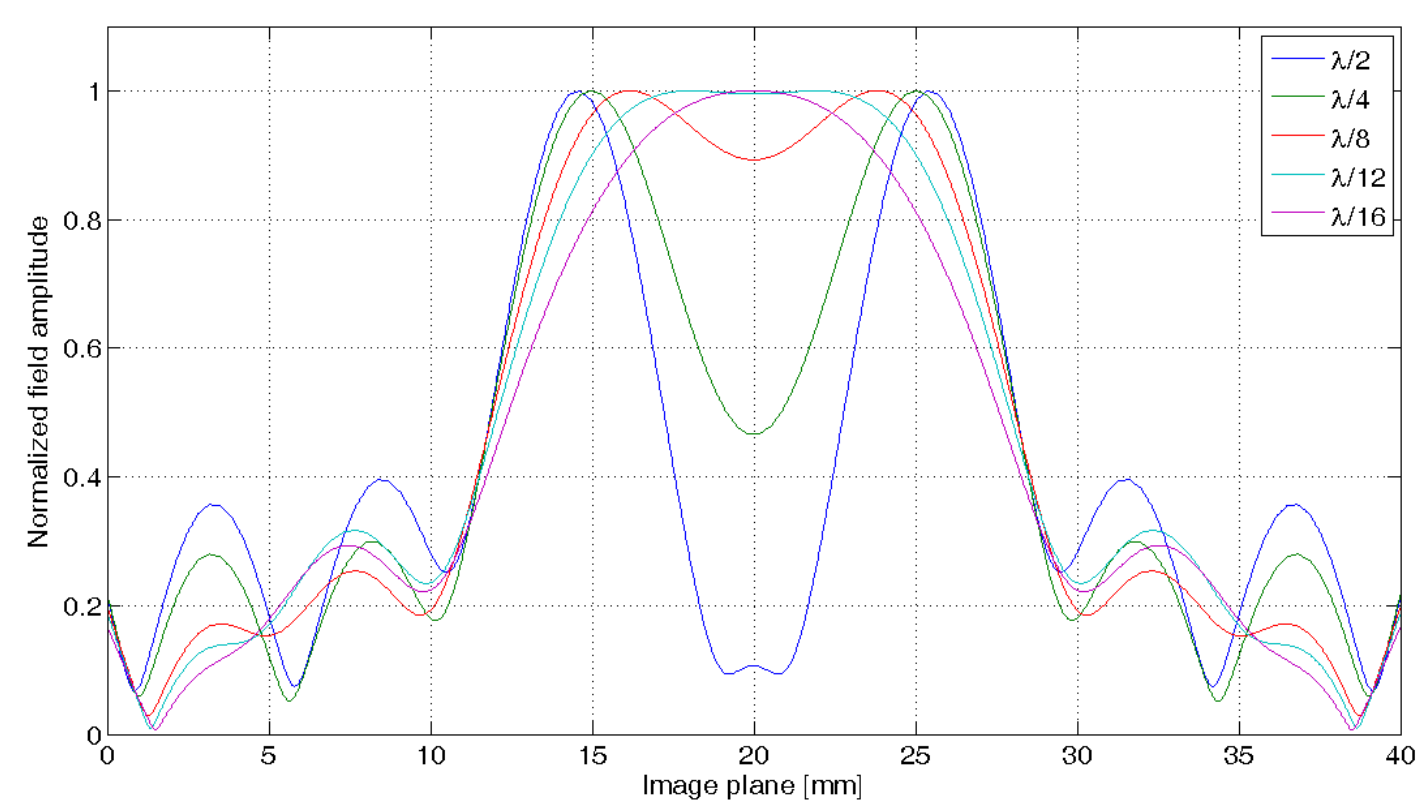

Fig. 14: Normalized electric field amplitude along the image plane.

\section{Conclusion}

A detailed simulation study using 3D metamaterial structures has been presented in this paper. The coupling between polarization independent cross-cylinder fishnet metamaterial layers has been investigated and a FOM of 45 at $39 \mathrm{GHz}$ has been demonstrated. This is seen as an improvement over current reached values in the literature. It is also worth mentioning that since the structure's period is $4.9 \mathrm{~mm} \approx 0.6^{*} \lambda$, this structure is not perfect for high sub wavelength imaging, whereas it can be considered as a good candidate for high transmission applications, for example as quasi-optical matching structures for automotive radomes [13].

\section{Acknowledgement}

This work is supported by a grant from Deutsche Forschungsgemeinschaft (DFG) under contracts No. BE 2256/11-1 and BE 2256/11-2.

\section{References}

[1] J.B. Pendry and D.R. Smith: "Reversing Light: Negative Refraction," American Institute of Physics (2003).

[2] X. Zhang and Z. Liu, "Superlenses to Overcome the Diffraction Limit," Nat. Mater., No. 6, Vol. 7, 435 - 441(2008).

[3] C.M. Soukoulis, M. Kafesaki, and E.N. Economou, "Negative-Index Materials: New Frontiers in Optics," Adv. Mater., Vol. 18, 1941-1952 (2006). 
[4] J.B. Pendry, "Negative Refraction Makes a Perfect Lens," Phys. Rev. Lett., Vol. 85, No. 18, 3966 - 3969 (2000).

[5] M. Kafesaki, I. Tsiapa, N. Katsarakis, T. Koschny, C.M. Soukoulis and E.N. Economou, "Left Handed Metamaterials: The Fishnet Structure and its Variations," Phys. Rev. B, Vol. 75 (2007).

[6] W. Sfar Zaoui, K. Chen, W. Vogel and M. Berroth, "Low Loss Broadband Polarization Independent Fishnet Negative Index Metamaterial at $40 \mathrm{GHz}$," Photon Nanostruct: Fundam. Appl (2011).

[7] X. Chen, T.M. Grzegorczyk, B.I. Wu, J. Pacheco, and J.A. Kong, "Robust Method to Retrieve the Constitutive Effective Parameters of Metamaterials," Phys. Rev. E, Stat. Phys. Plasmas Fluids Relat. Interdiscip. Top, Vol. 70 (2004).

[8] B.D.F. Casse, W.T. Lu, Y.J. Huang and S. Sridhar, "Robust Method to Determine the Resolution of a Superlens by Analyzing the Near-Field Image of a Two-Slit Object," Physics.optics, Vol. 1105 (2011).

[9] A. F. Starr, P. M. Rye, D. R. Smith and S. Nemat-Nasser, "Fabrication and Characterization of a Negative-Refractive-Index Composite Metamaterial," Phys. Rev. B, Vol. 70 (2004).

[10] K. Aydin, Z. Li, L. Sahin and E. Ozbay, "Negative Phase Advance in Polarization Independent Multi-Layer Negative-Index Metamaterials,” Opt. Express, Vol. 16, 8835 8844 (2008).

[11] J. Zhou, T. Koschny and C.M. Soukoulis, "An Efficient Way to Reduce Losses of LeftHanded Metamaterials," Opt. Express, Vol. 16, 11147-11152 (2008).

[12] Viktor A. Podolskiy, Nicholas A. Kuhta, and Graeme W. Milton, "Optimizing the Superlens: Manipulating Geometry to Enhance the Resolution," Appl. Phys. Lett. 87, 231113 (2005).

[13] F. Fitzek, Z. Abou-Chahine, R.H. Rasshofer, E.M. Biebl, "Automotive Radome DesignFishnet Structure for 79 GHz,” German Microwave Conference 2010, 147-149. 